\title{
A randomized phase II study of gemcitabine plus Z-360, a CCK2 receptor-selective antagonist, in patients with metastatic pancreatic cancer as compared with gemcitabine plus placebo
}

\author{
Makoto Ueno ${ }^{1}$ Chung Pin $\mathrm{Li}^{2,3} \cdot$ Masafumi Ikeda $^{4} \cdot$ Hiroshi Ishii $^{5,17} \cdot$ Nobumasa Mizuno $^{6}$ - Taketo Yamaguchi ${ }^{7}$. \\ Tatsuya Ioka $^{8,18} \cdot$ Do Youn Oh' • Wataru Ichikawa ${ }^{10} \cdot$ Takuji Okusaka $^{11} \cdot$ Yutaka Matsuyama $^{12} \cdot$ Daichi Arai $^{13}$. \\ Li Tzong Chen ${ }^{14} \cdot$ Young Suk Park ${ }^{15} \cdot$ Junji Furuse $^{16}$
}

Received: 8 May 2017 / Accepted: 1 June 2017 / Published online: 20 June 2017

(C) The Author(s) 2017. This article is an open access publication

\begin{abstract}
Background We investigated the efficacy and safety of 60 , 120 , or $240 \mathrm{mg}$ of Z-360, which is a highly potent cholecystokinin2-receptor-selective antagonist, combined with gemcitabine in patients with metastatic pancreatic cancer. Methods Patients were randomly assigned in a 1:1:1:1 ratio to one of four treatment groups. Patients received $1000 \mathrm{mg} /$ $\mathrm{m}^{2}$ gemcitabine for each cycle and Z-360 tablets of $60 \mathrm{mg}$ (GZ $60 \mathrm{mg}$ group), 120, $240 \mathrm{mg}$ or placebo tablets (Gem
\end{abstract}

Electronic supplementary material The online version of this article (doi:10.1007/s00280-017-3351-4) contains supplementary material, which is available to authorized users.

Makoto Ueno

uenom@kcch.jp

1 Division of Hepatobiliary and Pancreatic Medical Oncology, Kanagawa Cancer Center, 2-3-2, Nakao, Asahi-ku, Yokohama-shi, Kanagawa 241-8515, Japan

2 Division of Gastroenterology and Hepatology, Department of Medicine, Taipei Veterans General Hospital, 201, Section 2, Shin-Pai Road, Taipei 11217, Taiwan

3 School of Medicine, National Yang-Ming University, 155, Section 2, Linong Street, Taipei 112, Taiwan

4 Department of Hepatobiliary and Pancreatic Oncology, National Cancer Center Hospital East, 6-5-1, Kashiwanoha, Kashiwa-shi, Chiba 277-8577, Japan

5 Department of Gastroenterology, Cancer Institute Hospital, Japanese Foundation for Cancer Research, 3-8-31, Ariake, Koto-ku, Tokyo 135-8550, Japan

6 Department of Gastroenterology, Aichi Cancer Center Hospital, 1-1 Kanokoden, Chikusa-ku, Nagoya, Aichi 464-8681, Japan

7 Department of Gastroenterology, Chiba Cancer Center, 666-2 Nitona-cho, Chuo-ku, Chiba-shi, Chiba 260-8717, Japan group) orally twice daily. The primary endpoint was overall survival (OS).

Results The median OS was 1.3 months longer in the GZ $60 \mathrm{mg}$ group compared with the Gem group (8.5 vs. 7.2 months) and the risk of death was reduced by $19 \%$ compared with the Gem group, although there were no statistically significant differences. The study treatments were well tolerated.

Conclusions In this Phase II study, no statistically significant differences between the GZ groups and Gem group were detected in any analysis. However, Z-360 in dose of $60 \mathrm{mg}$ tends to improve OS in patients with metastatic pancreatic cancer with low toxic effect. Further exploratory

8 Department of Gastrointestinal Cancer Screening and Surveillance, Osaka Medical Center for Cancer and Cardiovascular Disease, 3-3 Nakamichi 1-Chome, Higashinari-ku, Osaka 537-8511, Japan

9 Division of Medical Oncology, Department of Internal Medicine, Seoul National University Hospital, Cancer Research Institute, Seoul National University College of Medicine, 101 Daehak-ro, Jongno-gu, Seoul 03080, South Korea

10 Division of Medical Oncology, Department of Medicine, Showa University School of Medicine, 1-30 Fujigaoka, Aoba-ku, Yokohama, Kanagawa 227-8501, Japan

11 Department of Hepatobiliary and Pancreatic Oncology, National Cancer Center Hospital, 5-1-1, Tsukiji, Chuo-ku, Tokyo 104-0045, Japan

12 Department of Biostatistics, School of Public Health, The University of Tokyo, 7-3-1 Hongo, Bunkyo-ku, Tokyo, Japan

13 Division of Clinical Research 3, ZERIA Pharmaceutical Co., Ltd., 10-11, Nihonbashi Kobuna-cho, Chuo-ku, Tokyo 103-8351, Japan 
trials with other agents such as gemcitabine plus nab-paclitaxel might be beneficial.

Keywords Clinical trial · Phase II - Pancreatic cancer · Metastatic $\cdot$ Gemcitabine $\cdot$ Cholecystokinin

\section{Introduction}

Pancreatic cancer (PC) is the eighth leading cause of cancer-related mortality worldwide [1], and the majority of patients do not have curable disease. Since 1997, gemcitabine therapy has been the standard first-line chemotherapy for patients with unresectable locally advanced or metastatic PC; however, 1-year survival rates of patients with metastatic disease are only approximately $20 \%[2,3]$. FOLFIRINOX has become one of the standard regimens for metastatic PC. However, the use of FOLFIRINOX is limited to patients in good medical condition because of high toxicity [2]. Gemcitabine plus nab-paclitaxel is another treatment option for patients in fair medical condition [3]. However, outcomes remain poor, and some patients cannot receive such intensive chemotherapy. There is thus an urgent need to develop other less toxic treatment options that have other mechanisms of action.

The gastrointestinal peptides gastrin and cholecystokinin (CCK) have been found to stimulate the growth of several human pancreatic cancer cell lines in culture and pancreatic xenograft rodent models [4]. Because the proliferation of PC cells has been reported to be mediated by CCK 2 receptor, this receptor has been suggested to be a potential therapeutic target for PC [5]. CCK-2 receptor downregulation can also enhance the apoptosis of pancreatic cancer cells [6].

Z-360 is a novel, orally active, and highly potent CCK2receptor-selective antagonist. Preclinical studies have

14 National Institute of Cancer Research, National Health Research Institutes, 367, Sheng-Li Rd., North District, 70456 Tainan, Taiwan

15 Division of Hematology-Oncology, Department of Medicine, Samsung Medical Center, Sungkyunkwan University School of Medicine, 50, Irwon-Dong, Gangnam-Gu, Seoul 06351, South Korea

16 Department of Internal Medicine, Medical Oncology, Kyorin University School of Medicine, 6-20-2, Shinkawa, Mitaka-shi, Tokyo 181-8611, Japan

17 Present Address: Department of Gastroenterology, Clinical Research Center, National Hospital Organization Shikoku Cancer Center, 160, Kou, Minamiumemoto-machi, Matsuyama-shi, Ehime 791-0280, Japan

18 Present Address: Department of Gastrointestinal Cancer Screening and Surveillance, Osaka International Cancer Institute, 3-1-69, Otemae, Chuo-ku, Osaka 541-8567, Japan shown that Z-360 either alone or in combination with gemcitabine can inhibit the growth of PC in animal xenograft models [7].

In a randomized phase Ib/IIa study, oral Z-360 at dose of 120 or $240 \mathrm{mg}$ twice daily combined with standard, weekly gemcitabine $1000 \mathrm{mg} / \mathrm{m}^{2}$ was well tolerated in patients with advanced PC. More patients receiving Z-360 reported improvement in pain than those receiving gemcitabine alone. In addition, patients who received Z-360 $120 \mathrm{mg}$ twice daily had better survival than those who received Z-360 $240 \mathrm{mg}$ twice daily or gemcitabine alone [8]. To confirm and extend the results of that study, we investigated the efficacy and safety of 60,120 , or $240 \mathrm{mg}$ of Z-360 twice daily combined with gemcitabine in this phase II study.

\section{Patients and methods}

\section{Study design}

This phase II multicenter, randomized, double-blind, parallel group, placebo-controlled study, sponsored by ZERIA Pharmaceutical Co., Ltd., Tokyo, Japan, was conducted at 27 sites in Japan, South Korea, and Taiwan in accordance with the Declaration of Helsinki and the International Conference on Harmonization Consolidated Guideline E6 for Good Clinical Practice. Written informed consent for participation in the study was obtained from all patients. Patients were randomly assigned in a 1:1:1:1 ratio to receive gemcitabine plus placebo (Gem group), Z-360 $60 \mathrm{mg}$ (GZ $60 \mathrm{mg}$ group), Z-360 $120 \mathrm{mg}$ (GZ $120 \mathrm{mg}$ group), or Z-360 $240 \mathrm{mg}$ (GZ $240 \mathrm{mg}$ group). Randomization was performed according to a computer-generated schedule and carried out by independent interactive web response system. Patients were dynamically assigned to the treatment groups in a stochastic manner. The allocations factors were the Eastern Cooperative Oncology Group (ECOG) performance status (PS) $(0+1$ vs. 2$)$, history of systemic chemotherapy for metastatic PC (yes vs. no), and site per country.

\section{Patients}

Eligible adults (20 years of age or older) had histological or cytological evidence of metastatic PC (either measurable or non-measurable disease according to the Response Evaluation Criteria in Solid Tumors [RECIST], version 1.1.), an ECOG PS 0-2, a life expectancy of $\geq 12$ weeks, and adequate hematologic, hepatic, and renal functions. Both chemo-naïve patients and patients who received any previous systemic chemotherapy (except gemcitabine) prior to 
4 weeks before randomization were eligible (Supplementary Text 1). Patient's eligibility was determined by each investigator.

\section{Treatment}

Patients received gemcitabine in a dose of $1000 \mathrm{mg} / \mathrm{m}^{2}$ as a 30-min intravenous infusion once weekly for 3 weeks of each 28-day cycle Dose interruption or reduction of gemcitabine was performed according to predefined criteria (Supplementary Text 2). Patients received Z-360 tablets in a fixed dose of 60,120 , or $240 \mathrm{mg}$ or matching placebo tablets orally twice daily until disease progression, unacceptable toxic effects, or the withdrawal of consent.

\section{Assessments}

Patient's conditions were assessed on each visit to receive gemcitabine. All adverse events (AEs) were evaluated according to the National Cancer Institute Common Terminology Criteria for Adverse Events, version 4.0. Tumor lesions were assessed by computed tomography or magnetic resonance imaging every 6 weeks until the treatment discontinuation criteria were met. The attending physicians radiologically assessed disease progression according to study-specific modified RECIST (Supplementary Text 3). Clinical disease progression was also assessed on the basis of the patient's global status. An independent review committee (IRC) assessed radiological disease progression according to RECIST, version 1.1. Progression-free survival (PFS) was calculated on the basis of the date of radiological disease progression as evaluated by the IRC. Clinical disease progression as evaluated by each investigator was also used as an event when calculating PFS.

\section{Endpoints}

The primary endpoint was overall survival (OS), defined as the time from the date of randomization to the date of death. If a patient was alive at the end of the study or was lost to follow-up, data on OS were censored. Secondary endpoints were PFS, response rate (RR), disease control rate (DCR), quality of life (QOL) score (Supplementary Text 4), and AEs.

\section{Statistical analysis}

The required sample size was estimated on the basis of the following assumptions: a hazard ratio (HR) of '1:0.70:0.70:0.70' (Gem group:GZ $60 \mathrm{mg}$ group:GZ $120 \mathrm{mg}$ group:GZ $240 \mathrm{mg}$ group), a 12-month recruitment period and a 12-month follow-up period, and a 30\% 1-year survival rate in the Gem group. At a significance level (one-sided) of $20 \%$, a Cox proportional-hazards model was used to compare the difference in survivor function between the Gem group and pooled GZ group (pooled data from the GZ $60 \mathrm{mg}$, GZ $120 \mathrm{mg}$, and GZ $240 \mathrm{mg}$ groups) by using a contrast ratio of $-1: 1 / 3: 1 / 3: 1 / 3$. A power of $81.6 \%$ was achieved according to the results of simulation analysis with 40 patients in each treatment group. It was estimated that 118 events would be needed to achieve the power.

The full analysis set (FAS) included randomized patients who received at least one dose of the study drug and for whom any information on post-baseline survival status was available. The safety set (SS) included all patients who received at least one dose of the study drug. The FAS was used for all efficacy evaluations and the SS was used for all safety evaluations.

Time-to-event variables such as OS, PFS, and survival rate and corresponding 2-sided 95\% CI for each treatment group were analyzed with the Kaplan-Meier method. The HR of time-to-event variables and the corresponding 2-sided $95 \%$ CI were determined with a Cox proportional hazards model including treatment group, ECOG PS $(0+1$ vs. 2), and previous treatment for metastatic PC (yes vs. no) as covariates, with a baseline hazard unique to each country. Subgroup analyses were performed without any adjustment.

The responses to the European Organization for Research and Treatment of Cancer (EORTC) Quality of Life Questionnaire Core 30 (QLQ-C30) questionnaires were analyzed in accordance with the EORTC guidelines [9]. Repeated-measures linear regression analysis was performed with the use of a mixed model to assess the QOL endpoint. The model used for the analysis of QOL included the explanatory variables of treatment group, baseline score, ECOG PS $(0+1$ vs. 2$)$, previous history of systemic chemotherapy for metastatic PC (yes vs. no), visit and country, and visit by treatment interaction. The analysis plan was finalized before breaking the key code, and the statistical analyses were performed at Zeria Pharmaceutical Co., Ltd. All statistical calculations were performed with the use of SAS, Release 9.3 (SAS Institute Inc., Cary, NC, USA).

\section{Results}

\section{Patients}

A total of 167 patients (95 in Japan, 44 in South Korea, 28 in Taiwan) were randomly assigned to treatment between April 2014 and November 2014. The data cut-off date was November 30, 2015. All randomized patients (41, 43, 42, and 41 patients in the GZ $60 \mathrm{mg}$, GZ $120 \mathrm{mg}$, GZ $240 \mathrm{mg}$, 


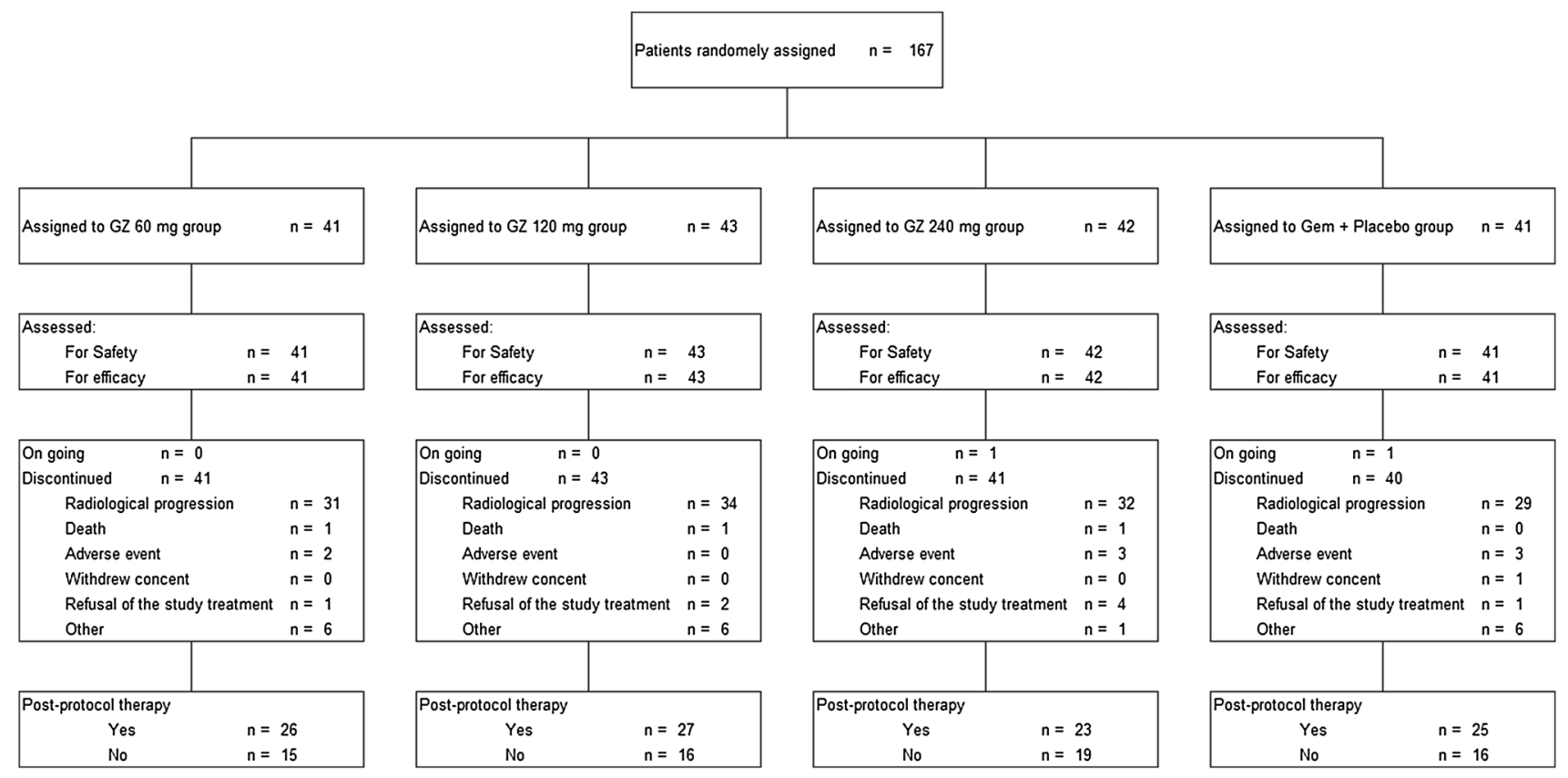

Fig. 1 CONSORT diagram

and Gem groups, respectively) were included in the FAS and SS populations (Fig. 1). No patient violated the eligibility criteria. Few patients in each group had a PS of 2 or a history of systemic chemotherapy for metastatic PC. The proportion of patients with a neutrophil-to-lymphocyte ratio (NLR) of $>4.0$, which was reported to be a prognostic factor in advanced PC [10], was higher in the GZ $240 \mathrm{mg}$ group than in the other groups (Table 1).

\section{Efficacy}

The number of events was 140 (84\% of patients). The median survival $(95 \% \mathrm{CI})$ was 8.5 months (5.8-9.8), 7.9 months (6.0-10.4), 6.8 months (4.1-8.4), and 7.2 months (5.4-8.8) in the GZ $60 \mathrm{mg}$, GZ $120 \mathrm{mg}$, GZ $240 \mathrm{mg}$, and Gem groups, respectively (Fig. 2a). The HR for OS $(95 \% \mathrm{CI})$ was $0.89(0.60-1.32)$ in the pooled Z-360 group and $0.81(0.50-1.32), 0.85(0.53-1.37)$, and 1.01 (0.62-1.64) in the GZ $60 \mathrm{mg}$, GZ $120 \mathrm{mg}$, and GZ $240 \mathrm{mg}$ groups, respectively. The efficacy of Z-360 in the subgroup of chemo-naïve metastatic PC was better than that of the overall patient population (HR in OS: $0.73,0.79$, and 0.85 in the GZ $60 \mathrm{mg}$, GZ $120 \mathrm{mg}$, and GZ $240 \mathrm{mg}$ groups, respectively) (Fig. 2c; Supplementary Table 1). The 1-year survival rate $(95 \% \mathrm{CI})$ was $23.4 \%(11.8-37.3), 23.1 \%$ (11.6-36.8), $18.6 \%$ (8.3-32.1), and 20.5\% (9.7-34.3), in the GZ $60 \mathrm{mg}$, GZ $120 \mathrm{mg}$, GZ $240 \mathrm{mg}$, and Gem groups, respectively.

In the analysis of PFS, 161 patients $(96.4 \%)$ had disease progression or died. The median PFS $(95 \% \mathrm{CI})$ was
3.9 months (1.7-5.6), 2.8 months (1.4-3.9), 2.9 months (1.6-4.1), and 2.6 months (1.4-4.0) in the GZ $60 \mathrm{mg}$, GZ $120 \mathrm{mg}$, GZ $240 \mathrm{mg}$, and Gem groups, respectively (Fig. 2b). The HR for PFS (95\% CI) was $0.82(0.56-1.20)$ in the pooled Z-360 group, and $0.74(0.47-1.16), 0.87$ (0.55-1.39), and $0.85(0.54-1.36)$ in the GZ $60 \mathrm{mg}$, GZ $120 \mathrm{mg}$, and GZ $240 \mathrm{mg}$ groups, respectively.

Post-protocol anticancer therapy was given to $26(63 \%)$, $27(63 \%), 23(55 \%)$, and $25(61 \%)$ patients in the GZ $60 \mathrm{mg}$, GZ $120 \mathrm{mg}$, GZ $240 \mathrm{mg}$, and Gem groups, respectively. The most common therapy was $\mathrm{S}-1$ (27\% in total). Only a few patients received FOLFIRINOX (3\% in total) or gemcitabine plus nab-paclitaxel ( $8 \%$ in total) as post-protocol anticancer therapy.

The response rates $(95 \% \mathrm{CI})$ according to independent review were $9.8 \%(2.7-23.1 \%), 11.6 \%$ (3.9-25.1\%), $9.5 \%$ (2.7-22.6\%), and 2.4\% (0.1-12.9\%), in the GZ $60 \mathrm{mg}$, GZ $120 \mathrm{mg}$, GZ $240 \mathrm{mg}$, and Gem groups, respectively. There were no major differences in DCR among the groups. In pre-specified subgroup analysis, the efficacy of Z-360 in the patients with chemo-naïve metastatic PC was better than that in the group as a whole (Fig. 2c; Supplementary Table 1).

Baseline carbohydrate antigen 19-9 (CA19-9) levels were measured in 166 patients. A total of 59, 58, 38, and $34 \%$ of the patients had a reduction of $\geq 20 \%$ from the baseline level in the GZ $60 \mathrm{mg}$, GZ $120 \mathrm{mg}$, GZ $240 \mathrm{mg}$, and Gem groups, respectively.

The responses to the QLQ-C30 questionnaire at baseline were evaluated for all patients in the FAS 
Table 1 Patient characteristics (full analysis set)

\begin{tabular}{lllll}
\hline Character- & GZ 60 mg & GZ 120 mg & GZ 240 mg \\
istic & $(N=41)$ & $(N=43)$ & $\begin{array}{l}\text { Gem + pla- } \\
\text { cebo }\end{array}$ \\
& $n(\%)$ & $n(\%)$ & $n(\%)$ & $(N=41)$ \\
& & & & $n(\%)$ \\
\hline
\end{tabular}

\begin{tabular}{|c|c|c|c|c|}
\hline \multicolumn{5}{|l|}{ Age (years) } \\
\hline Mean & 63.7 & 66.7 & 65.2 & 64.6 \\
\hline Median & 66.0 & 68.0 & 67.0 & 65.0 \\
\hline $\begin{array}{l}<65 \\
\quad \text { (years) }\end{array}$ & $19(46.3)$ & $16(37.2)$ & $18(42.9)$ & $19(46.3)$ \\
\hline $\begin{array}{l}\geq 65 \\
\quad \text { (years) }\end{array}$ & $22(53.7)$ & $27(62.8)$ & $24(57.1)$ & $22(53.7)$ \\
\hline \multicolumn{5}{|l|}{ Gender } \\
\hline Male & $26(63.4)$ & $24(55.8)$ & $24(57.1)$ & $22(53.7)$ \\
\hline Female & $15(36.6)$ & $19(44.2)$ & $18(42.9)$ & $19(46.3)$ \\
\hline \multicolumn{5}{|c|}{ Body surface area $\left(\mathrm{m}^{2}\right)$} \\
\hline Mean & 1.589 & 1.553 & 1.531 & 1.569 \\
\hline Median & 1.548 & 1.553 & 1.575 & 1.579 \\
\hline \multicolumn{5}{|c|}{ Eastern Cooperative Oncology Group Performance Status ${ }^{\mathrm{a}}$} \\
\hline 0 & $19(46.3)$ & $18(41.9)$ & $21(50.0)$ & $22(53.7)$ \\
\hline 1 & $22(53.7)$ & $22(51.2)$ & $19(45.2)$ & $18(43.9)$ \\
\hline 2 & $0(0.0)$ & $3(7.0)$ & $2(4.8)$ & $1(2.4)$ \\
\hline \multicolumn{5}{|c|}{ CA19-9 level ${ }^{\mathrm{b}}$} \\
\hline Mean & 5682.7 & 6693.6 & 6704.5 & 5013.2 \\
\hline Median & 669.0 & 682.6 & 614.4 & 349.6 \\
\hline \multicolumn{5}{|c|}{ Neutrophil lymphocyte ratio } \\
\hline$\leq 4$ & $32(78.0)$ & $35(81.4)$ & $24(57.1)$ & $29(72.5)$ \\
\hline$>4$ & $9(22.0)$ & $8(18.6)$ & $18(42.9)$ & $11(27.5)$ \\
\hline \multicolumn{5}{|c|}{ Previous treatment for metastatic PA } \\
\hline Yes & $5(12.2)$ & $7(16.3)$ & $5(11.9)$ & $5(12.2)$ \\
\hline No & $36(87.8)$ & $36(83.7)$ & $37(88.1)$ & $36(87.8)$ \\
\hline \multicolumn{5}{|c|}{ Diagnostic information } \\
\hline $\begin{array}{l}\text { Initial } \\
\text { occur- } \\
\text { rence }\end{array}$ & $36(87.8)$ & $35(81.4)$ & $32(76.2)$ & $33(80.5)$ \\
\hline $\begin{array}{l}\text { Reoccur- } \\
\text { rence }\end{array}$ & $5(12.2)$ & $8(18.6)$ & $10(23.8)$ & $8(19.5)$ \\
\hline
\end{tabular}

a Eastern Cooperative Oncology Group Performance Status range from 0 to 4, with lower scores indicating better performance status

b A total of 166 patients had a baseline CA19-9 measurement. 1 patient in Gem + placebo group was excluded from the analysis due to missing data

population. The scores for "Global Health Status and Quality of Life scale" and "scale for pain" deteriorated at week 4 in all groups, but at week 12 both scales recovered to the same level as that at baseline in GZ $60 \mathrm{mg}$ and GZ $120 \mathrm{mg}$ groups. In contrast, both scales deteriorated in a similar manner in the GZ $240 \mathrm{mg}$ and Gem groups (Supplementary Table 2). No major differences were noted among the groups in the other variables.

\section{Safety}

Only 4 treatment-related serious adverse events (SAEs) were reported: cellulitis in the GZ $60 \mathrm{mg}$ group, upper gastrointestinal hemorrhage and Pneumocystis jirovecii pneumonia in the GZ $240 \mathrm{mg}$ group, and vomiting in the Gem group. Fatal events, excluding progression of PC, were pulmonary embolism in the GZ $60 \mathrm{mg}$ group, acute respiratory failure in the GZ $120 \mathrm{mg}$ group, and pneumonia aspiration, cardiac failure, and small intestinal obstruction in the GZ $240 \mathrm{mg}$ group. The proportion of patients with SAEs or with AEs leading to discontinuation of the study treatment did not differ among the groups. AEs that occurred in more than $10 \%$ of patients in any treatment group are shown in Table 2. Hematological events known as adverse reactions of gemcitabine, such as decreased neutrophil count were reported more often in the GZ $60 \mathrm{mg}$ and GZ $120 \mathrm{mg}$ groups than in the other groups. However, no febrile neutropenia was reported in any group. In contrast, fatigue, asthenia, and decreased appetite were reported more often in the GZ $240 \mathrm{mg}$ group than in the other groups.

High exposure to the study drug and high relative dose intensity (RDI) (>90\%) of gemcitabine were maintained in all treatment groups.

\section{Discussion}

In this multicenter, placebo-controlled, randomized, phase II study, no statistically significant differences between the GZ groups and Gem group were detected in any analysis. However, Z-360 tends to improve OS and PFS in patients with metastatic PC.

The median OS and PFS were prolonged by 1.3 months in the GZ $60 \mathrm{mg}$ group as compared with the respective values in the Gem group. In the GZ $60 \mathrm{mg}$ group, a $19 \%$ reduction in the risk of death, and a $26 \%$ reduction in the risk of disease progression or death as compared with the Gem group were observed. Higher proportions of patients in the GZ $60 \mathrm{mg}$ and GZ $120 \mathrm{mg}$ groups had reductions of $\geq 20 \%$ in serum CA19-9 levels, which has been reported to be associated with superior survival [11], as compared with the gemcitabine group, and reduced CA19-9 secretion might indicate cytotoxicity associated with cell apoptosis. This result was similar to that in a pivotal study of gemcitabine plus nab-paclitaxel (proportions of patients who had reductions of $\geq 20 \%$ in serum CA19-9 levels: $61 \%$ [gemcitabine plus nab-paclitaxel group] versus $44 \%$ [gemcitabine group]) [3].

The study treatments were well tolerated, and high exposure to the study drug and high RDI ( $>90 \%)$ of gemcitabine 
A

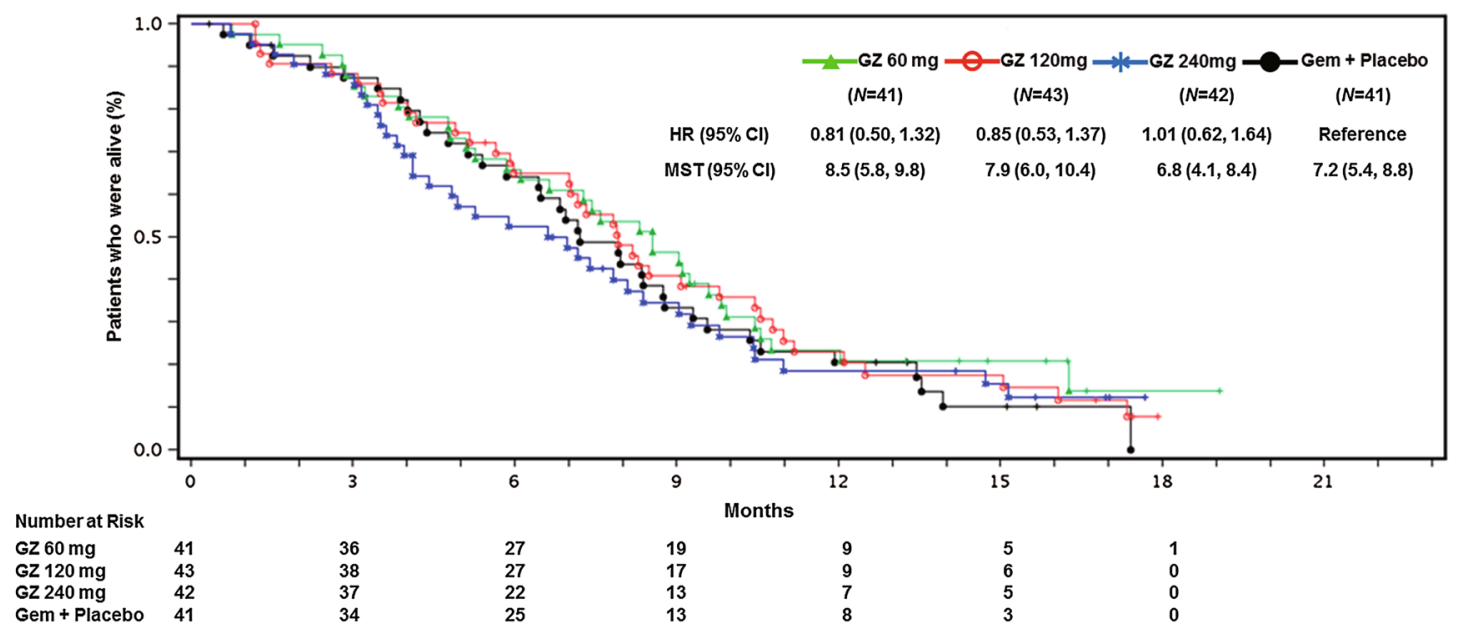

B

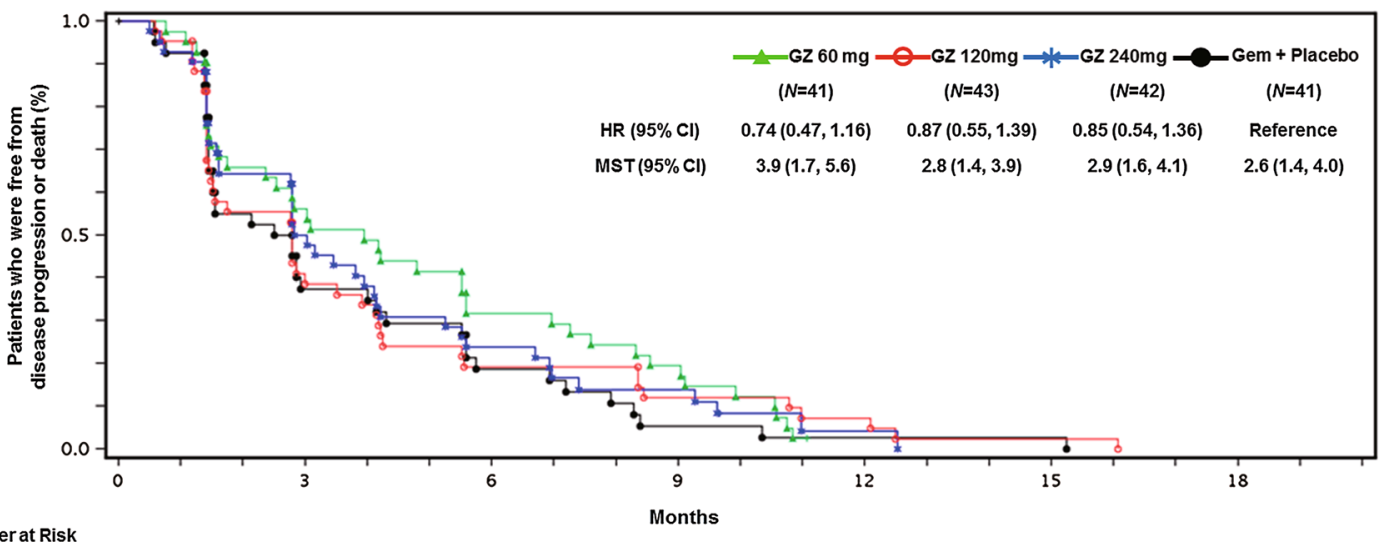

$\begin{array}{lllrlll}\text { Numberat Risk } & & & & 3 & \\ \text { GZ 60 mg } & 41 & 21 & 13 & 9 & 2 & 0 \\ \text { GZ 120 mg } & 43 & 27 & 13 & 3 & 1 & 1 \\ \text { GZ 240 mg } & 42 & 16 & 8 & 4 & 1 & 1 \\ \text { Gem+ Placebo } & 41 & 17 & 7 & & \end{array}$

C

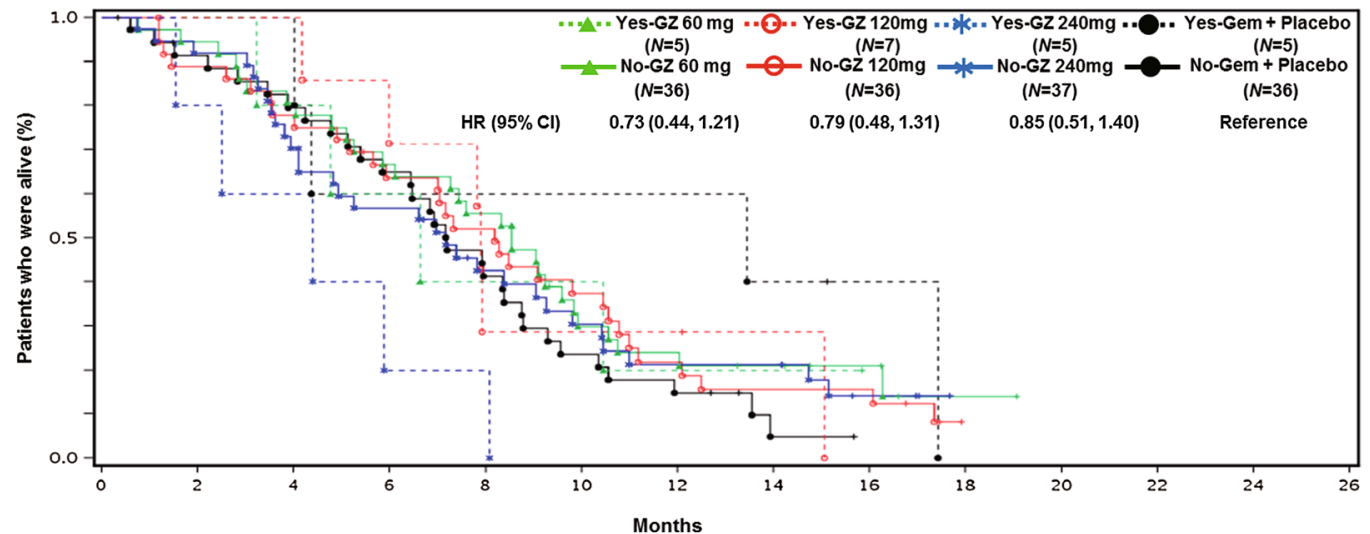

Fig. 2 Kaplan-Meier estimates of a overall survival, b progression-free survival (assessed by an independent review committee) and $\mathbf{c}$ overall survival in a subgroup analysis of previous treatment for metastatic PC (yes vs. no) (full analysis set) 
Table 2 Treatment-emergent adverse events reported by $\geq 10 \%$ of patients in any treatment group and receipt of immunostimulants (safety set)

\begin{tabular}{|c|c|c|c|c|}
\hline & $\begin{array}{l}\mathrm{GZ} 60 \mathrm{mg} \\
(N=41) \\
n(\%)\end{array}$ & $\begin{array}{l}\mathrm{GZ} 120 \mathrm{mg} \\
(N=43) \\
n(\%)\end{array}$ & $\begin{array}{l}\mathrm{GZ} 240 \mathrm{mg} \\
(N=42) \\
n(\%)\end{array}$ & $\begin{array}{l}\text { Gem }+ \text { placebo } \\
(N=41) \\
n(\%)\end{array}$ \\
\hline \multicolumn{5}{|l|}{ Hematological analysis } \\
\hline Platelet count decreased & $17(41.5)$ & $20(46.5)$ & $10(23.8)$ & $11(26.8)$ \\
\hline Neutrophil count decreased & $21(51.2)$ & $13(30.2)$ & $14(33.3)$ & $9(22.0)$ \\
\hline$\geq$ Grade 3 & $16(39.0)$ & $12(27.9)$ & $13(31.0)$ & $9(22.0)$ \\
\hline Neutropenia & $2(4.9)$ & $5(11.6)$ & $2(4.8)$ & $3(7.3)$ \\
\hline$\geq$ Grade 3 & $2(4.9)$ & $4(9.3)$ & $2(4.8)$ & $2(4.9)$ \\
\hline White blood cell count decreased & $13(31.7)$ & $14(32.6)$ & $10(23.8)$ & $7(17.1)$ \\
\hline Anemia & $16(39.0)$ & $15(34.9)$ & $7(16.7)$ & $12(29.3)$ \\
\hline Receipt of immunostimulants & $3(7.3)$ & $2(4.7)$ & $3(7.1)$ & $4(9.8)$ \\
\hline \multicolumn{5}{|l|}{ Non-hematological analysis } \\
\hline Weight decreased & $6(14.6)$ & $4(9.3)$ & $1(2.4)$ & $0(0.0)$ \\
\hline Nausea & $16(39.0)$ & $19(44.2)$ & $15(35.7)$ & $15(36.6)$ \\
\hline Constipation & $17(41.5)$ & $11(25.6)$ & $8(19.0)$ & $10(24.4)$ \\
\hline Vomiting & $10(24.4)$ & $10(23.3)$ & $8(19.0)$ & $11(26.8)$ \\
\hline Diarrhea & $4(9.8)$ & $16(37.2)$ & $6(14.3)$ & $6(14.6)$ \\
\hline Ascites & $4(9.8)$ & $8(18.6)$ & $5(11.9)$ & $3(7.3)$ \\
\hline Stomatitis & $5(12.2)$ & $3(7.0)$ & $1(2.4)$ & $4(9.8)$ \\
\hline Pyrexia & $12(29.3)$ & $10(23.3)$ & $9(21.4)$ & $12(29.3)$ \\
\hline Fatigue & $8(19.5)$ & $5(11.6)$ & $11(26.2)$ & $8(19.5)$ \\
\hline Edema peripheral & $6(14.6)$ & $8(18.6)$ & $5(11.9)$ & $2(4.9)$ \\
\hline Malaise & $3(7.3)$ & $6(14.0)$ & $2(4.8)$ & $8(19.5)$ \\
\hline Asthenia & $3(7.3)$ & $2(4.7)$ & $6(14.3)$ & $3(7.3)$ \\
\hline Decreased appetite & $16(39.0)$ & $19(44.2)$ & $22(52.4)$ & $9(22.0)$ \\
\hline Hypoalbuminemia & $3(7.3)$ & $5(11.6)$ & $4(9.5)$ & $1(2.4)$ \\
\hline Rash & $2(4.9)$ & $5(11.6)$ & $7(16.7)$ & $6(14.6)$ \\
\hline Rash maculo-papular & $3(7.3)$ & $2(4.7)$ & $2(4.8)$ & $5(12.2)$ \\
\hline Nasopharyngitis & $2(4.9)$ & $1(2.3)$ & $5(11.9)$ & $0(0.0)$ \\
\hline Cancer pain & $3(7.3)$ & $3(7.0)$ & $5(11.9)$ & $3(7.3)$ \\
\hline Insomnia & $5(12.2)$ & $4(9.3)$ & $4(9.5)$ & $3(7.3)$ \\
\hline
\end{tabular}

were maintained in all treatment groups. Therefore, this treatment is considered quite manageable.

Gemcitabine plus Z-360 was associated with few safety concerns as compared with FOLFIRINOX or gemcitabine plus nab-paclitaxel. For example, the proportions of patients with a decrease in neutrophil count/neutropenia $\geq$ Grade 3 in each GZ group in this study (Table 2) were low as compared with the results of a pivotal study in Japan of gemcitabine plus nab-paclitaxel (neutropenia: $70.6 \%$ ) [12]. In addition, no febrile neutropenia was reported in any GZ group (gemcitabine plus nab-paclitaxel: $5.9 \%$ ).

Moreover, the scores on the "Global Health Status and Quality of Life scale" and "scales for pain" at 12 weeks, reported to be important scales for the evaluation of $\mathrm{PC}$ [13], were maintained at the baseline levels in the GZ $60 \mathrm{mg}$ and GZ $120 \mathrm{mg}$ groups.
Z-360 thus can be easily given to patients who cannot tolerate highly cytotoxic treatment or who prefer to maintain their QOL.

The efficacy of Z-360 in subgroup of chemo-naïve metastatic PC was better than that of the overall patient population, which should be considered when conducting other trials in the future.

The OS in the GZ $60 \mathrm{mg}$ and GZ $120 \mathrm{mg}$ groups was better than that in the GZ $240 \mathrm{mg}$ group, although the results for PFS were similar among the Z-360 groups. Higher proportion of patients has shown an NLR of $>4.0$ in GZ $240 \mathrm{mg}$ group. AEs related to the patient's global status, such as fatigue, asthenia, and decreased appetite, were reported more often in the GZ $240 \mathrm{mg}$ group than in the other groups, which might have led to difficulty in administering subsequent therapies. Such factors might have resulted in the difference between OS and PFS. 
Although our study had limitations because of its small sample size and exploratory nature, our results suggest that the efficacy of Z-360 might have reached a plateau at $60 \mathrm{mg}$ twice daily. Because Z-360 is attributed to a different mechanism of action from that of currently available cytotoxic agents, it might also be beneficial to combine Z-360 with other agents, such as gemcitabine plus nab-paclitaxel. Recently, a CCK2 receptor-selective antagonist combined with an immune checkpoint inhibitor was reported to be effective in mice [14].

In conclusion, Z-360 tends to improve OS and PFS with low toxic effect in patients with metastatic PC. Further exploratory trials of $60 \mathrm{mg} \mathrm{Z-360} \mathrm{twice} \mathrm{daily} \mathrm{with} \mathrm{other}$ agents might be beneficial.

Acknowledgements We thank the patients, their families, and the all investigators who participated in the study (detailed in the supplementary Appendix) for their contributions.

Funding support This work was supported by Zeria Pharmaceutical Co., Ltd.

\section{Compliance with ethical standards}

Conflict of interest Makoto Ueno reports Grants and personal fees from Zeria Pharmaceutical, during the conduct of the study; Grants and personal fees from Taiho, AstraZeneca, Grants from Daiichi Sankyo, Eisai, Ono, Nobelpharma, personal fees from Eli lilly. Yakult, Novartis, outside the submitted work; Masafumi Ikeda reports Grants from Zeria Pharmaceutical, during the conduct of the study; Grants and personal fees from Bayer Yakuhin, Bristol Myers, Eisai, Taiho Pharmaceutical, Eli Lilly Japan, Chugai Pharmaceutical, Yakult, Kyowa Hakko Kirin, Nano Carrier, Grants from Boehringer Ingelheim, Ono Pharmaceutical, AstraZeneca, Baxter, Merck Serono, Kowa, Cimic, personal fees from Novartis Pharma, Abbott Japan, Nippon Kayaku, Daiichi Sankyo, outside the submitted work; Hiroshi Ishii reports Grants from Zeria Pharmaceutical, during the conduct of the study; Grants and personal fees from Taiho Pharmaceutical, personal fees from Nobelpharma, Mochida Pharmaceutical, Hospira Japan, Ono Pharmaceutical, outside the submitted work; Nobumasa Mizuno reports Grants from Zeria Pharmaceutical, during the conduct of the study; Grants and personal fees from Taiho Pharmaceutical, Grants from Merck Serono, AstraZeneca, NanoCarrier, Eisai, MSD, personal fees from Novartis, Pfizer, Kyowa Hakko Kirin, Yakult Honsha, Eli Lilly Japan K.K., outside the submitted work; Tatsuya Ioka reports Grants from Zeria Pharmaceutical, during the conduct of the study; Grants and personal fees from AstraZeneca, Taiho, Grants from Merk Serono, Nihon Zouki, Bayer, Glaxo SmithKline, Mundipharma, personal fees from Chugai, Yakult Honsha, JCRO, Eisai, Mochida, Fuji Film Pharm, Daiichi Sankyo, Nihon Kayaku, outside the submitted work; Do Youn Oh reports Grants from Zeria Pharmaceutical, during the conduct of the study; Grants and personal fees from AstraZeneca, Grants from Lilly, personal fees from Merck, outside the submitted work; Wataru Ichikawa reports personal fees from Zeria Pharma, during the conduct of the study; Grants and personal fees from Taiho, Merck Serono, Chugai Pharma, Takeda, Ono Pharma, Grants from Eisai, personal fees from Bayer, Daiichi Sankyo, outside the submitted work; Takuji Okusaka reports Grants and personal fees from Zeria Pharmaceutical., during the conduct of the study; Grants and personal fees from Novartis Pharma K.K., Taiho Pharmaceutical, Merck Serono, Eli Lilly Japan K.K., Dainippon Sumitomo Pharma, Bayer Yakuhin., Yakult Honsha, Nobelpharma, Baxter, AstraZeneca K.K., Ono Pharmaceutical, Nip- pon Boehringer Ingelheim, Grants from Eisai, Kowa Company, Kyowa Hakko Kirin, Pfizer Japan, Glaxo SmithKline K.K., Shizuoka Industry, Nano Carrier, Chugai Pharmaceutical, personal fees from Nippon kayaku, Astellas Pharma, FUJIFILM RI Pharma, EA Pharma, Nippon Chemiphar, Daiichi Sankyo, Celgene, K.K., outside the submitted work; Yutaka Matsuyama reports personal fees from STATCOM, outside the submitted work; Daichi Arai is employee of Zeria Pharmaceutical; Junji Furuse reports Grants and personal fees from Zeria Pharmaceutical, during the conduct of the study; Grants and personal fees from Taiho Pharmaceutical, Chugai Pharmaceutical, Yakult, Kyowa Hakko Kirin, Eli Lilly Japan, Ono Pharmaceutical, Bayer Pharmaceutical, Merck Serono, Novartis, J-Pharma, Takeda Pharmaceutical, Daiichi Sankyo, Astellas Pharma, Bristol-Myers Squibb, Nippon Kayaku, Sumitomo Dainippon Pharma, Mochida Pharma, Shionogi, Grants from Onco Therapy Science, Glaxo SmithKline, Torii Pharma, Janssen Pharmaceutical, Sanofi, Hisamitsu Pharmaceutical, Pfizer, personal fees from Eisai, Fujifilm, Otsuka Pharmaceutical, Boehringer Ingelheim, AstraZeneca, Mitsubishi Tanabe Pharma, MSD, Meiji Seika Pharma, Ajinomoto Pharmaceuticals, Sawai, Sandoz, outside the submitted work; Chung Pin Li, Taketo Yamaguchi and Young Suk Park reports Grants from Zeria Pharmaceutical, during the conduct of the study; Li Tzong Chen has nothing to disclose.

Ethical approval All procedures performed in studies involving human participants were in accordance with the ethical standards of the institutional and/or national research committee and with the 1964 Helsinki declaration and its later amendments or comparable ethical standards.

Informed consent Informed consent was obtained from all individual participants included in the study.

Open Access This article is distributed under the terms of the Creative Commons Attribution 4.0 International License (http://creativecommons.org/licenses/by/4.0/), which permits unrestricted use, distribution, and reproduction in any medium, provided you give appropriate credit to the original author(s) and the source, provide a link to the Creative Commons license, and indicate if changes were made.

\section{References}

1. Jemal A, Bray F, Center MM, Ferlay J, Ward E, Forman D (2011) Global cancer statistics. CA Cancer J Clin 61(2):69-90

2. Conroy T, Desseigne F, Ychou M, Bouché O, Guimbaud R, Bécouarn Y, Adenis A, Raoul J-L, Gourgou-Bourgade S, Cdl Fouchardière, Bennouna J, Bachet J-B, Khemissa-Akouz F, Péré-Vergé D, Delbaldo C, Assenat E, Chauffert B, Michel P, Montoto-Grillot C, Chem M, Ducreux M (2011) FOLFIRINOX versus gemcitabine for metastatic pancreatic cancer. N Engl J Med 364:1817-1825

3. Von-Hoff DD, Ervin T, Arena FP, Chiorean EG, Infante J, Moore M, Seay T, Tjulandin SA, Ma WW, Saleh MN, Harris M, Reni M, Dowden S, Laheru D, Bahary N, Ramanathan RK, Tabernero J, Hidalgo M, Goldstein D, Van-Cutsem E, Wei X, Iglesias J, Renschler MF (2013) Increased survival in pancreatic cancer with nab-paclitaxel plus gemcitabine. N Engl J Med 369(18):1691-1703

4. Clerc P, Leung-Theung-Long S, Wang TC, Dockray GJ, Bouisson M, Delisle M-B, Vaysse N, Pradayrol L, Fourmy D, Dufresne M (2002) Expression of CCK2 receptors in the murine pancreas: proliferation, transdifferentiation of acinar cells, and neoplasia. Gastroenterology 122(2):428-437 
5. Rengifo-Cam W, Singh P (2004) Role of progastrins and gastrins and their receptors in GI and pancreatic cancers: targets for treatment. Curr Pharm Des 10(19):2345-2358

6. Fino KK, Matters GL, McGovern CO, Gilius EL, Smith JP (2012) Downregulation of the CCK-B receptor in pancreatic cancer cells blocks proliferation and promotes apoptosis. Am J Physiol Gastrointest Liver Physiol 302(11):G1244-G1252

7. Kawasaki D, Emori Y, Eta R, Iino Y, Hamano H, Yoshinaga K, Tanaka T, Takei M, Watson SA (2008) Effect of Z-360, a novel orally active CCK-2/gastrin receptor antagonist on tumor growth in human pancreatic adenocarcinoma cell lines in vivo and mode of action determinations in vitro. Cancer Chemother Pharmacol 61:883-892

8. Meyer T, Caplin ME, Palmer DH, Valle JW, Larvin M, Waters JS, Coxon F, Borbath I, Peeters M, Nagano E, Kato H (2009) A phase Ib/IIa trial to evaluate the CCK2 receptor antagonist Z-360 in combination with gemcitabine in patients with advanced pancreatic cancer. Eur J Cancer 46(3):526-533

9. Fayers P, Aaronson N, Bjordal K, Groenvold M, Curran D, Bottomley A (2001) EORTC QLQ-C30 scoring manual. European Organisation for Research and Treatment of Cancer, Brussels

10. Vivaldi C, Caparello C, Musettini G, Pasquini G, Catanese S, Fornaro L, Lencioni M, Falcone A, Vasile E (2016) First-line treatment with FOLFOXIRI for advanced pancreatic cancer in clinical practice: patients' outcome and analysis of prognostic factors. J Cancer Clin Trials 139(4):938-945

11. Maisey NR, Norman AR, Hill A, Massey A, Oates J, Cunningham D (2005) CA19-9 as a prognostic factor in inoperable pancreatic cancer: the implication for clinical trials. Br J Cancer 93(7):740-743

12. Ueno H, Ikeda M, Ueno M, Mizuno N, Ioka T, Omuro Y, Nakajima TE, Furuse J (2016) Phase I/II study of nab-paclitaxel plus gemcitabine for chemotherapy-naive Japanese patients with metastatic pancreatic cancer. Cancer Chemother Pharmacol 77(3):595-603

13. Fayers P, Weeden S, Curran D (1998) EORTC QLQ-C30 reference values. European Organisation for Research and Treatment of Cancer, Brussels

14. Smith JP, Wang S, Reham A, Jablonski SA, Weiner LM (2016) Combination therapy with immune checkpoint inhibitor and CCK-receptor blockade increases survival of pancreatic cancer [abstract]. In: Proceedings of the 107th Annual Meeting of the American Association for Cancer Research (AACR), New Orleans, Louisiana, vol 76 (14 Suppl). AACR, p Abstract nr 4023 